\title{
A view from the Internet age: Let's build a health system that meets the needs of the next generation
}

\section{Alejandro R. Jadad}

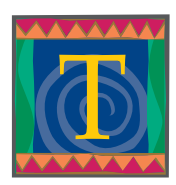

hree years ago, my youngest daughter, who was 7 years old at the time, discovered a mole on her leg. Terrified, she called me and said, "Dad, run and get our camera, take a picture of this spot and e-mail it to one of your doctor-friends to see if it's cancer." I replied, "I cannot do this." She looked shocked and asked, "Why not?" I looked at her and could not find a sensible answer. Earlier that day she had sent digital pictures to my mother, who lives in South America. It was embarrassing. And it continues to be so.

Over the past decade, we have witnessed the dramatic transformation of major industries by information and communication technologies (ICTs). Banking, entertainment and tourism will probably never be the same now that online consumer services have been introduced. Although new diagnostic and therapeutic technologies are used enthusiastically to support clinical activities, ICTs have had little effect on the health system, to the point that a patient today will notice little difference in how health care is delivered compared with 10 or even 20 years ago. (I use "health system" instead of "health care system" deliberately: we must move beyond our current disease-centric model to one that also promotes health.)

Now that the future of the health system is being hotly debated and major transfers of funds are expected throughout Canada to fix health care for a generation, ${ }^{1}$ we must avoid political bickering and start thinking about ways to ensure that the new investments translate into a health system that matches the reality of our next generation. This will be a challenge. Although current decision-makers witnessed the birth of the personal computer, mobile telephones, the Internet and the World Wide Web, they have so far overlooked the potential of these tools to transform the health system. It would be tragic if our children grow up to find that our efforts to sustain the system relied mainly on putting more resources into an outdated, industrial-age model of health care delivery and that, instead of an integrated, efficient and flexible system, we created one that looks more like a big, dysfunctional collection of repair shop franchises. ${ }^{2}$

I propose 4 ways in which ICTs could bring the current system closer to the reality of the next generation, using technology that we have available now ${ }^{3}$ and current trends in Internet use by Canadians. ${ }^{4}$ This list is by no means exhaustive. It includes:

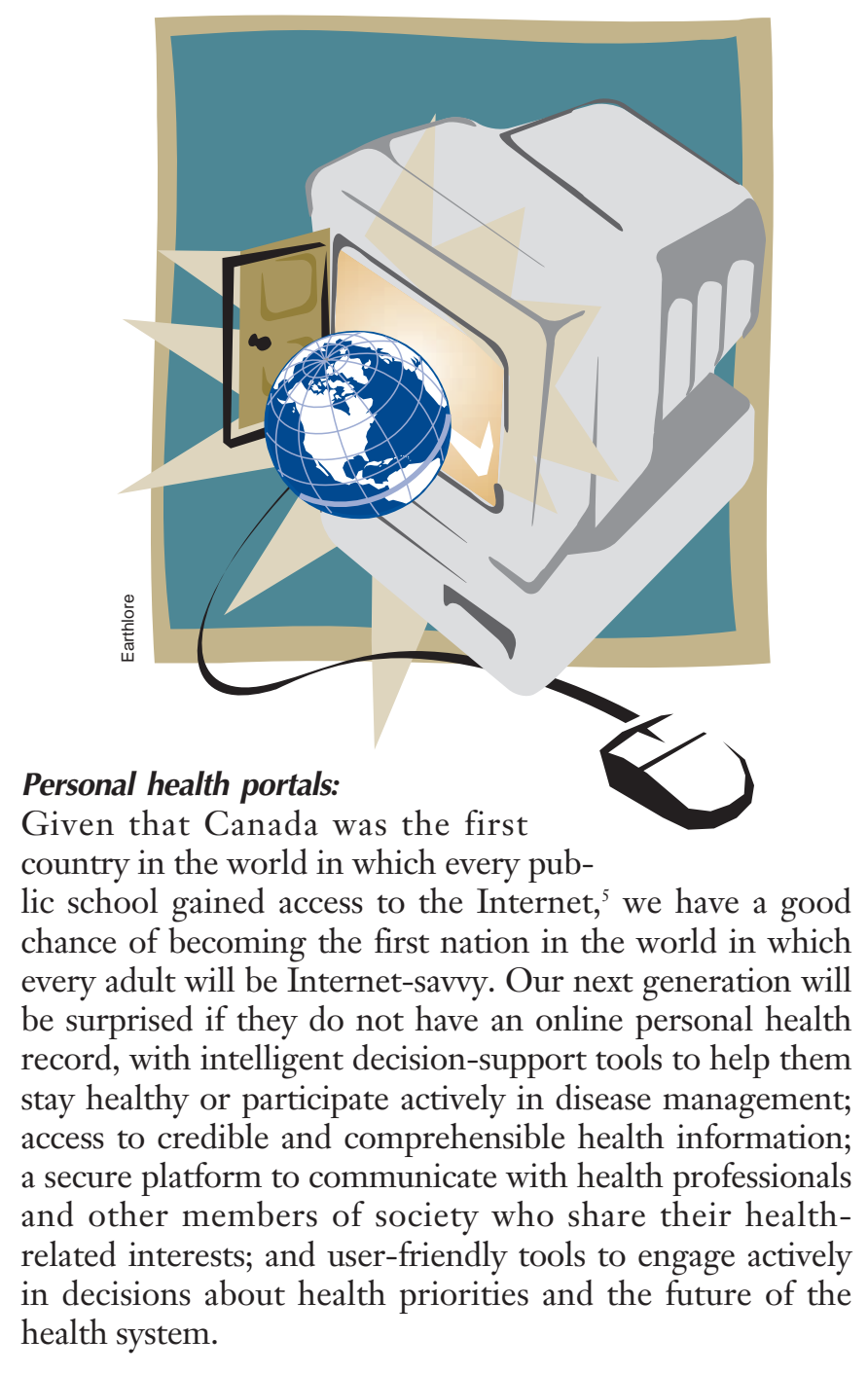

An integrated platform to enable coordinated access to health services: Thanks to online travel services, most Canadians can now book a flight, rent a car and reserve a hotel room, all on a single Web site and from any location. If fiercely competitive companies are able to support multivendor online services, it is reasonable for Canadians to expect that health organizations (which in theory should not be competing with each other) get their act together and support joint platforms to promote continuity and coordination of services. Our main risk at this point is that each province will create a separate 
health services platform that is incompatible with the others. This could create difficulties for the exchange of information across geographic boundaries and for the development of pan-Canadian efforts to optimize our ability to deliver and access high-quality health services with limited resources.

Telehealth services as an integral part of the system: Canada is already a world leader in telehealth. However, here, as in most other regions of the world, telehealth services consist mainly of live consultations that require participants to interact from dedicated, specialized facilities. Telehealth has thus remained a complementary service devoted to giving underserved areas access to specialized services. It could be argued that this approach reaffirms the industrial-age model of care. With the widespread penetration of the Internet, the reduction in cost of data storage and the proliferation of digital cameras, new modalities of telehealth are now possible. Today, patients who own Web cameras could request routine consultations with health professionals from home. Telephones with cameras are making multimedia teleconsultations possible from anywhere. By using digital cameras and any kind of Internet connection, it is also possible for patients and health professionals to capture clinically important images (e.g., moles, surgical wounds, electrocardiograms) from any location and send them to specialists in distant sites for assessment. This modality, known as asynchronous or store-and-forward telehealth, could have important effects on wait times and promote truly equitable access to health services, since specialists would be able to use their time more efficiently while serving anyone who needs them, from anywhere in Canada or beyond.
Effective incentives for the rapid adoption and use of ICTs: Our generation missed the opportunities created by the telephone: this ubiquitous and familiar tool remains underused because of the lack of financial incentives to use it within the health system. We will be repeating history unless we develop the financial, political, legal and logistic frameworks necessary to enable expedient identification, evaluation, introduction and adoption of new and powerful ICTs that could improve health and health care delivery.

Our children often wonder how we managed to go through school without the Internet. Let's make sure that, in 10 years, they thank us for using it to create the health system they expect and deserve.

Alejandro Jadad is the Director of the Centre for Global eHealth Innovation at the University Health Network and the University of Toronto, Toronto, Ont.

\section{References}

1. Liberal Party of Canada. A fix for a generation: The Paul Martin plan for better health care. Available: www.cbc.ca/canadavotes/pdfplatforms/healthcare en.pdf (accessed 10 Nov 2004).

2. Jadad AR. Quo vadis health system? On wishes, magic and the power of eHealth. Healthcare Info Manage Commun 2001;25(2):8. Available: http://hcccinc .qualitygroup.com/hcccinc2/pdf/Vol_XV_No_2/Vol_XV_No_2.html (accessed 10 Nov 2004).

3. Jadad AR, Delamothe T. What next for electronic communication and health care? BMF 2004;328:1143-4. Available: http://bmj.bmjjournals.com/cgi/ content/full/328/7449/1143 (accessed 10 Nov 2004).

4. Lupea D, Rizo CA, Anderson M, Baybourdy H, Closson T, Jadad A. What services would patients like to have available through the Internet when hospitals are disabled by an epidemic? Lessons from the SARS outbreak at the University Health Network in Toronto. Medinfo 2004 (CD):1733.

5. Industry Canada. Canada's SchoolNet. Available: http://schoolnetrescol.ic.gc. ca/e/whatis.htm (accessed 10 Nov 2004). 